Research Paper

\title{
Massive parallel sequencing and digital gene expression analysis reveals potential mechanisms to overcome therapy resistance in pulmonary neuroendocrine tumors
}

\author{
Robert Fred Henry Walter ${ }^{1,2}$, Claudia Vollbrecht ${ }^{3}$, Daniel Christoph ${ }^{4}$, Robert Werner ${ }^{5}$, Jan Schmeller2, \\ Elena Flom ${ }^{1,2}$, Georgia Trakada 6 , Aggeliki Rapti ${ }^{7}$, Vasilis Adamidis ${ }^{8}$, Wolfgang Hohenforst-Schmidt ${ }^{9}$, Jens \\ Kollmeier ${ }^{5}$, Thomas Mairinger ${ }^{5}$, Jeremias Wohlschlaeger ${ }^{2}$, Paul Zarogoulidis ${ }^{\natural}{ }^{凶}$, Konstantinos Porpodis ${ }^{8}$, \\ Kurt Werner Schmid², Fabian Dominik Mairinger ${ }^{2}$ \\ 1. Ruhrlandklinik, West German Lung Center, University Hospital Essen, University of Duisburg-Essen. \\ 2. Institute of Pathology, University Hospital Essen, University of Duisburg-Essen. \\ 3. Institute of Pathology, Charité Universitaetsmedizin, Berlin. \\ 4. Department of Oncology, University Hospital Essen, University of Duisburg-Essen. \\ 5. Institute of Pathology, Helios Klinikum Emil von Behring, Berlin. \\ 6. Division of Pulmonology, Department of Clinical Therapeutics, National and Kapodistrian University of Athens School of Medicine, Alexandra Hospital, \\ Athens, Greece. \\ 7. 2nd Department of Pulmonary Medicine, "Sotiria" Hospital of Chest Diseases, Athens, Greece. \\ 8. Pulmonary Department-Oncology Unit, "G. Papanikolaou" General Hospital, Aristotle University of Thessaloniki, Thessaloniki, Greece. \\ 9. Medical Clinic I,"Fuerth" Hospital, University of Erlangen, Fuerth, Germany. \\ $\square$ Corresponding author: Paul Zarogoulidis, M.D, Ph. D., Pulmonary Department-Oncology Unit, "G. Papanikolaou” General Hospital, Aristotle University of \\ Thessaloniki, Thessaloniki, Greece. Fax: 00302310992424; Mobile: 00306977271974; E-mail: pzarog@hotmail.com.
}

(1) Ivyspring International Publisher. Reproduction is permitted for personal, noncommercial use, provided that the article is in whole, unmodified, and properly cited. See http://ivyspring.com/terms for terms and conditions.

Received: 2016.07.21; Accepted: 2016.09.18; Published: 2016.10.25

\begin{abstract}
Background: Lung cancer is the leading cause of cancer-related deaths worldwide. $25 \%$ show neuroendocrine differentiation (typical/atypical carcinoids, large-/small-cell neuroendocrine carcinomas). Carcinoids present with long survival rates, but metastatic carcinoids correlate with decreased survival and are commonly insensitive to standard chemotherapy or radiation. Therefore, novel therapeutic strategies are urgently needed.

Material and methods: 70 representative tumor specimens were used for next-generation sequencing analysis of 14 genes related to therapy response. Additionally, mRNA-expression profiles of 60 matching samples were determined for 13 selected drug targets by using the NanoString nCounter technology.

Results: A number of features known to sensitize tumors for different targeted therapies could be identified, which hopefully improve the clinical management of this subgroup of lung neoplasias. In particular, EGFR expression was observed in the investigated tumors in a noteworthy manner. Additionally, MDM2 was strongly expressed in the majority of all samples whereas the expression of its physiological inhibitor, CDKN2A, was nearly absent in all low-grade tumors. TP53 showed a high frequency of variants in high-grade tumors but mutations were rare in carcinoids.

Conclusion: Based on our results, therapeutic approaches with MDM2-inhibitors and monoclonal anti-EGFR antibodies may be promising in pulmonary carcinoid tumors.
\end{abstract}

Key words: NanoString, next-generation sequencing, biomarkers, personalized therapy, lung cancer. 


\section{INTRODUCTION}

Lung cancer is the leading cause of cancer-related deaths worldwide [1-3], twenty-five percent of these belong to the group of neuroendocrine tumors [4]. These tumors encompass small-cell lung carcinoma (SCLC), large-cell neuroendocrine carcinoma (LCNEC), as well as typical (TC) and atypical carcinoids (AC) [5]. Patients suffering from TC have excellent survival rates with $87-100 \%$, but some present with lymph-node metastasis (4-15\%) [6-15]. Atypical carcinoids are more aggressive (survival rate $61-88 \%$ ) and show higher frequency of nodal metastasis [8-14, 16]. According to the WHO classification from 1999, LCNEC was assigned to the non-small cell lung cancers (NSCLC) but shows similar biological behavior as SCLC with a five-year survival rate of $15 \%-57 \%$ [4, 8]. Up to $20 \%$ of all lung cancer incidences are SCLC showing the poorest five-year survival rates with less than $5 \%[4,5,8,15]$. High-grade neuroendocrine carcinomas of the lung occur almost exclusively in older patients (median 61 years) with a history of smoking, whereas lung carcinoids occur frequently in never smokers and younger patients (mean 45-55 years) [7, 15, 17, 18].

Carcinoid tumors are predominantly treated surgically, metastatic tumors are commonly not sensitive to chemotherapeutic regimes or radiation [4, 19]. Adjuvant chemo- or radiotherapy should be considered in completely resected AC with mediastinal lymph node involvement [6, 20, 21]. Combinations of chemotherapeutics are usually platinum- or streptozoticin-based [20].

For SCLC, chemotherapy with cisplatin plus etoposide is a well-established treatment since these tumors are notably sensitive to chemo- and radiotherapy [4]. An optimal treatment for LCNEC is still under investigation due to the relative rarity of this entity [4]. As defined by the WHO classification guidelines, LCNEC belongs to NSCLC, leaving it to the physician whether to treat it similarly to SCLC or NSCLC [8]. In fact, patients receiving SCLC-based regimens showed a significantly better outcome [20, 22].

The present study was conducted to identify markers for personalized therapeutic concepts in pulmonary neuroendocrine tumors, potentially leading to an improved clinical management.

\section{MATERIAL AND METHODS}

\section{Demographic Data and Study Design}

The study is based on a collective of seventy representative formalin-fixed, paraffin-embedded pulmonary neuroendocrine tumors $(17 \mathrm{TC}, 17 \mathrm{AC}, 19$
LCNEC, and 17 SCLC) used for sequencing. For expression analysis, 60 samples were investigated (16 TC and 13 AC, 16 LCNEC and 15 SCLC). The initial diagnosis was re-evaluated by two experienced pathologists (JWO, TMA). Specimens were taken from the Institute of Pathology at the University Hospital Essen (Germany) from 2005 till 2012. TNM-staging was based on the WHO Classification of Tumours guidelines (2004) [18]. The mean age at date of diagnosis was 58.6 years (median age: 59.0 years; $95 \%$ CI: 50.8-66.9 months). Survival data were available for 34 patients with twenty-two reported deaths at the time of data collection. Patients receiving chemotherapy before resection were excluded. The study was approved by the ethical committee of the University Hospital Essen (ID: 13-5382-BO). The investigations conform to the principles outlined in the declaration of Helsinki.

\section{Sample Preparation}

Genomic DNA was isolated on a Maxwell ${ }^{\circledR} 16$ Research (Promega Corporation, Madison, USA) as recommended in the manufacturer's protocol. RNA extraction was performed using the RNeasy FFPE kit (Qiagen, Hilden, Germany) according to the manufacturer's recommendations. Nucleic acid quantification was performed using Qubit (Life Technologies, Carlsbad, USA) and Nanodrop 1000 instrument (Thermo Fisher Scientific, Waltham, USA). RNA integrity was assessed using an Agilent 2100 Bioanalyzer (Agilent Technologies, Santa Clara, USA) at the NanoString nCounter Core Facility at the University of Heidelberg (Germany) by smear analysis.

\section{NanoString CodeSet Design and Expression Quantification}

Important genes for different tumor-associated signaling pathways were included in the Custom CodeSet using the Standard Chemistry. The CodeSet contained a total of 91 genes and 13 of these genes were considered as potential pharmaceutical targets (ALK, CDKN2A, EGFR, FAS, FGFR1, FIGF, FLT4, IGF1, IGF2, KDR, MDM2, MET, MTOR).

Probe-sets for each gene were designed and synthesized at NanoString Technologies (Seattle, USA). Total RNA (100 ng) from FFPE material was measured at the NanoString nCounter Core Facility at the University of Heidelberg, Germany.

\section{Next-Generation Sequencing}

Sample preparation was done using the TruSeq Amplicon - Cancer Panel (Illumina Inc., San Diego, CA, USA) followed by paired-end sequencing on the MiSeq Personal Sequencer (Illumina) according the protocols provided by the manufacturer. The Cancer 
Panel covered the following 48 tumor-relevant genes (table 1) including 221 mutation hotspots. Library construction followed the TrueSeq Custom Amplicon - Library Preparation guide.

FastQ-files were aligned against the Hg19 build. For analysis of the aligned reads including variant calling, the software Avadis NGS (Strand Scientific Intelligence, California, USA) was used. Reads with a quality score $<30$ were discarded. After removal of SNPs, data filtering was done by excluding variants with $<25$ effective variant reads or below $10 \%$ variation frequency. Synonymous variants were removed.

Finally, variants were analyzed for their functional impact on the protein-activity by using MutationAssessor (release 2) [23] and implementation of the ANNOVAR algorithm [24], combining the tools SIFT [25], PolyPhen2 [26] and MutationTaster [27].

\section{Statistical Analysis}

All statistical analyses were performed using the $\mathrm{R}$ statistical programming environment (v3.1.3). For dichotomous factors and linear vectors the Wilcoxon Mann-Whitney rank sum test was applied. For variance analysis of variables with more than two categories the Kruskal-Wallis test was performed. Correlations between linear vectors were analyzed by Spearman's rank correlation test. Double dichotomous contingency tables were analyzed using Fisher's Exact test. To test dependency of ranked parameters with more than two groups the Pearson's Chi-squared test was used. COXPH-model was used to analyze overall survival (OS) and progression-free survival (PFS). PFS was calculated from the first day of chemotherapy until progression, death from any cause, or the last time of follow up. OS was defined as the time from diagnosis until the date of death or last follow-up. Surveillance of PFS and OS was stopped on August 31, 2014. Significant differences in PFS or OS were analyzed by the Wald-test, likelihood-ratio test and Score (logrank) test.

The level of statistical significance was defined as $\mathrm{p} \leq 0.05$.

\section{RESULTS}

\section{Gene Expression in Pulmonary Neuroendocrine Tumors}

Gene expression analysis showed strong differences between tumors of the four different entities, but also within tumors of each subgroup. Most strikingly, EGFR gene expression is present in $87.5 \%$ of TC, $100 \%$ of AC, $65.3 \%$ of LCNEC and in $60 \%$ of SCLC. Likewise, FGFR1 is expressed in $97.3 \%$ of all carcinoids in a noteworthy manner. Besides, $62.5 \%$ of LCNEC and $80 \%$ of SCLC show strong expression of FGFR1. MDM2 was strongly expressed in the majority of all samples. $81.3 \%$ of TC, $76.9 \%$ of AC, $50 \%$ of LCNEC and $93.4 \%$ of SCLC present gene expression of MDM2. CDKN2A expression was rare in all low-grade neuroendocrine lung tumors, but present in some carcinomas showing high gene expression.

An overview of cases with increased expression levels is given in figure 1 , an overview of all expression pattern is shown in table 2 .

\section{Occurrence of Mutations in Pulmonary Neuroendocrine Tumors}

86 functionally deleterious variants were determined within 13 therapy-relevant genes in 49 out of 70 samples. Four of the variants could be detected in TC, 14 in AC, 30 in LCNEC and 38 in SCLC.

In seven samples $(10 \%$; one $\mathrm{TC}$, two $\mathrm{AC}$, one LCNEC and three SCLC), variants in the EGFR gene were found, but none of them is known or predicted to activate the receptor. For $E R B B 2$, a receptor tyrosine kinase (RTK), closely related to EGFR, three different variants could be observed in eleven samples.

Activating KRAS variants downstream of these receptors were found in three tumors (two LCNEC and one AC). Additionally, one sample showed activating mutations in the NRAS gene locus. BRAF variants occurred in three samples $(4 \%)$, two LCNEC and one SCLC. Five different variants of PIK3CA were found in six tumor samples.

Table 1. Overview of all genes covered by the NGS cancer hotspot panel. The Panel consists of two-times 221 probes for paired-end sequencing covering 221 mutational hotspots in 48 genes.

\begin{tabular}{lllllll}
\hline ABL1 & AKT1 & ALK & APC & ATM & BRAF & CDHN2A \\
\hline CSF1R & CTNNB1 & EGFR & ERBB2 & ERBB4 & FBXW7 & FGFR1 \\
FGFR3 & FLT3 & GNA11 & GNAQ & GNAS & HNF1A & HRAS \\
JAK2 & JAK3 & KDR & KIT & KRAS & MET & IDH1 \\
NOTCH1 & NPM1 & NRAS & PDGFRA & PIK3CA & PTEN & MPL \\
RET & SMAD4 & SMARCB1 & SMO & SRC & STK11 & RB1 \\
\hline
\end{tabular}


Table 2. Results of the gene expression analysis. Minimum, maximum and median for all 13 therapy relevant markers in the overall cohort of pulmonary neuroendocrine tumours is shown. Additionally, the mean value of tumours expressing the mentioned markers as well as the percentage of tumours expressing these markers is shown for each entity. Besides, the number of cases showing an outstanding expression level, including minimum and maximum value, is listed.

\begin{tabular}{|c|c|c|c|c|c|c|c|c|c|c|c|c|c|c|}
\hline \multirow{2}{*}{ Target } & \multicolumn{3}{|c|}{ Overall Cohort } & \multicolumn{2}{|c|}{ TC } & \multicolumn{2}{|c|}{$A C$} & \multicolumn{2}{|c|}{ LCNEC } & \multicolumn{2}{|c|}{ SCLC } & \multicolumn{3}{|c|}{ Cases with Outstanding Expression } \\
\hline & $\min$ & median & $\max$ & mean* & $\%^{* *}$ & mean* & $\%^{* *}$ & mean* & $\%^{* *}$ & mean $^{*}$ & $\% * *$ & amount & $\min$ & $\max$ \\
\hline ALK & 0 & 0 & 1216 & 215 & $12,50 \%$ & 729 & $15,38 \%$ & - & $0,00 \%$ & 1023 & $6,67 \%$ & 2 & 1023 & 1216 \\
\hline EGFR & 0 & 447 & 6729 & 1903 & $87,50 \%$ & 1520 & $100,00 \%$ & 1569 & $56,25 \%$ & 662 & $60,00 \%$ & 18 & 1131 & 6729 \\
\hline FAS & 0 & 0 & 5017 & 293 & $62,50 \%$ & 448 & $23,08 \%$ & 1842 & $31,25 \%$ & 445 & $20,00 \%$ & 3 & 1031 & 5017 \\
\hline FGFR1 & 0 & 1106 & 8048 & 1808 & $100,00 \%$ & 2708 & $92,31 \%$ & 2382 & $62,50 \%$ & 1154 & $80,00 \%$ & 18 & 2099 & 8048 \\
\hline FIGF & 0 & 0 & 3896 & 489 & $37,50 \%$ & - & $0,00 \%$ & 1393 & $31,25 \%$ & 660 & $6,67 \%$ & 5 & 731 & 3896 \\
\hline FLT4 & 0 & 33 & 1518 & 490 & $68,75 \%$ & 400 & $38,46 \%$ & 202 & $12,50 \%$ & 266 & $20,00 \%$ & 6 & 574 & 1518 \\
\hline IGF1 & 0 & 107 & 7262 & 509 & $87,50 \%$ & 670 & $38,46 \%$ & 2067 & $56,25 \%$ & 360 & $53,33 \%$ & 7 & 1306 & 7262 \\
\hline IGF2 & 0 & 382 & 39203 & 29429 & $87,50 \%$ & 22787 & $84,62 \%$ & 2262 & $56,25 \%$ & 1334 & $86,67 \%$ & 18 & 1737 & 39203 \\
\hline KDR & 0 & 99 & 6139 & 901 & $68,75 \%$ & 848 & $53,85 \%$ & 2233 & $31,25 \%$ & 550 & $40,00 \%$ & 13 & 1001 & 6139 \\
\hline MDM2 & 0 & 1170 & 7054 & 2201 & $81,25 \%$ & 1516 & $76,92 \%$ & 2235 & $50,00 \%$ & 2236 & $93,33 \%$ & 33 & 1099 & 7054 \\
\hline MET & 0 & 145 & 4475 & 485 & $68,75 \%$ & 960 & $61,54 \%$ & 1551 & $31,25 \%$ & 583 & $60,00 \%$ & 8 & 913 & 4475 \\
\hline MTOR & 0 & 588 & 4250 & 856 & $81,25 \%$ & 664 & $100,00 \%$ & 1646 & $43,75 \%$ & 750 & $80,00 \%$ & 13 & 991 & 4250 \\
\hline & & & & & & & & & & & & & & \\
\hline & & & & & & & & & & & & & & \\
\hline & & & & & & & & & & & & & & \\
\hline & & & & & & & & & & & & & & \\
\hline * ...M Me & xpressi & on of mark & express & g tumour & & & & & & & & & & \\
\hline$* * \ldots \%=$ & rcentag & of tumou & expressi & ig the mar & ker & & & & & & & & & \\
\hline ... all $v$ & s are $\mathrm{N}$ & anoString & ounter m & RNA coun & & & & & & & & & & \\
\hline
\end{tabular}

\% Cases with Outstanding Expression

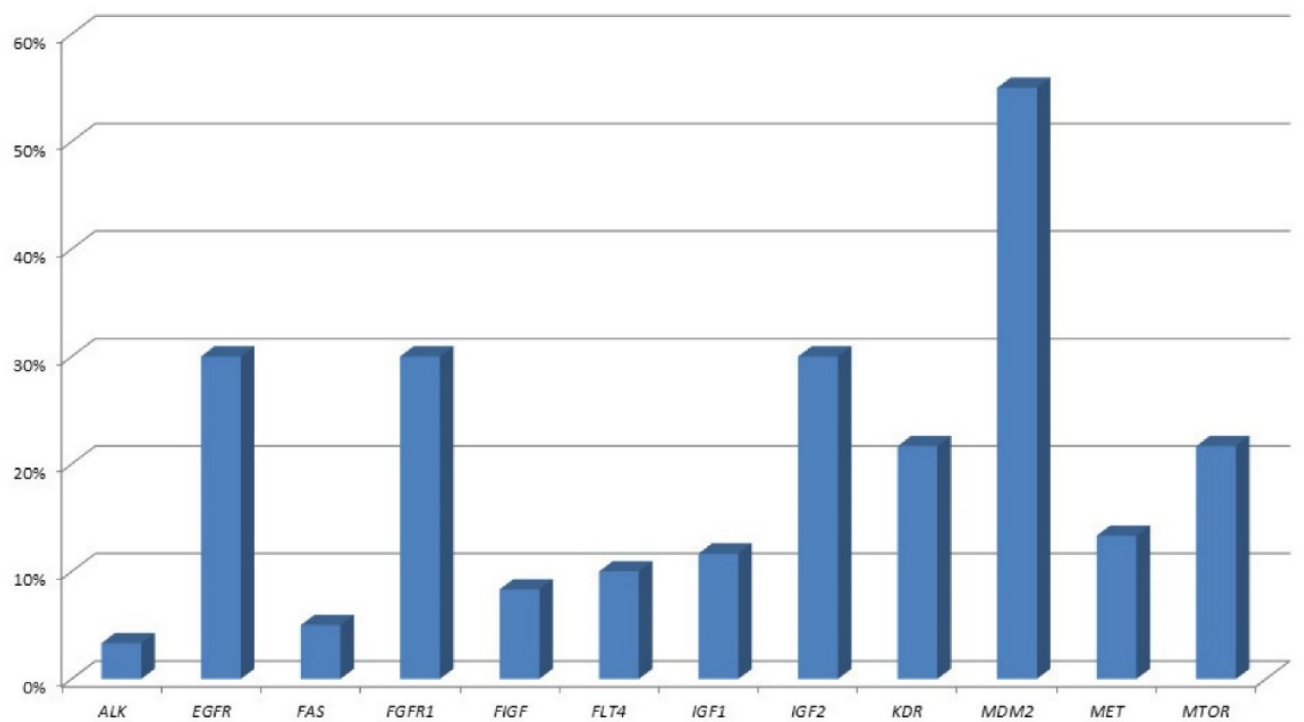

Figure 1. Percentage of tumours showing highly increased gene expression. One-third of all pulmonary neuroendocrine tumours show strong overexpression of EGFR and FGFRI. Of note, also one-third of tumours show high IGF2 expression and $10 \%$ present with high IGFI expression. More than $50 \%$ of all tumours show highly elevated MDM2 expression.

Interestingly, TP53 showed the highest frequency of variants with 31 alterations in 23 samples $(33 \%)$, whereas the percentage was varying in the different neuroendocrine subtypes. $64.7 \%$ of all SCLC and $63.2 \%$ LCNEC showed a functional inactivation of P53 via TP53 mutations. No TP53 alterations were found in TC or in AC.

A summary of all mutations is given in Supplementary table 1.

Expression and mutation frequency are summarized in table 3 for each tumor entity.

\section{Association with Clinicopathological Parameters}

EGFR, FLT4 and FIGF show highly significant differences in their expression levels between the four investigated tumor entities $(p=0.0044, p=0.0054$ and $\mathrm{p}=0.0096$, respectively). Elevated EGFR gene expression $(p=0.0002)$ is more prominent in carcinoid tumors compared to carcinomas. Also FGFR1 gene expression presents with significant differences $(\mathrm{p}=0.0301)$. Furthermore, upregulated EGFR gene expression associated significantly with lower 
IASLC-Grade ( $\mathrm{p}=0.0005)$. FGFR1 as well as FLT4 show highly significant associations with tumor grade ( $p=0.0025$ and $p=0.0061$, respectively). High FIGF $(\mathrm{p}=0.0210$, HR: 1.40) and FAS ( $\mathrm{p}=0.0233$, HR: 1.27) gene expression levels seem to be associated with shortened PFS. In high-grade tumors, high FIGF mRNA levels identified a group of poor responders to therapy (COXPH: $\mathrm{p}=0.0347)$.

The appearance of TP53 variants was significantly associated with tumor type $(\mathrm{p}<0.0001)$ and is associated with carcinomas. PIK3CA shows variants in $5.9 \%$ of $\mathrm{AC}$ and $23.5 \%$ of SCLC, whereas TC and LCNEC present none of these variants $(p=0.0116)$. Tumors infiltrating lymph nodes present with higher frequency of TP53 variants $(p=0.0007)$, as well as $E R B B 2$ variants $(\mathrm{p}=0.0051)$ or $B R A F$ mutations $(\mathrm{p}=0.0468)$.

PIK3CA variants are associated with shortened OS ( $\mathrm{p}=0.0019$, HR: 2.54). Activating mutations in the KRAS gene ( $\mathrm{p}=0.0003, \mathrm{HR}:>10)$ are associated with a higher risk of progression.

Table 3. Overview of results of the biomarker screening. Data for the percentage of tumours expressing each marker as well as the percentage of tumours showing inactivating genetic variants are shown.

\begin{tabular}{|c|c|c|c|c|c|c|c|c|}
\hline BRAF & & $0 \%$ & & $0 \%$ & & $11 \%$ & & $6 \%$ \\
\hline EGFR & $88 \%$ & $6 \%$ & $100 \%$ & $12 \%$ & $56 \%$ & $6 \%$ & $60 \%$ & $18 \%$ \\
\hline ERBB2 & & $12 \%$ & & $12 \%$ & & $16 \%$ & & $24 \%$ \\
\hline FAS & $63 \%$ & & $23 \%$ & & $31 \%$ & & $20 \%$ & \\
\hline FGFR1 & $100 \%$ & $0 \%$ & $92 \%$ & $6 \%$ & $63 \%$ & $5 \%$ & $80 \%$ & $0 \%$ \\
\hline FIGF & $38 \%$ & & $0 \%$ & & $31 \%$ & & $7 \%$ & \\
\hline FLT4 & $69 \%$ & & $38 \%$ & & $13 \%$ & & $20 \%$ & \\
\hline HRAS & & $0 \%$ & & $0 \%$ & & $0 \%$ & & $0 \%$ \\
\hline IGF1 & $88 \%$ & & $38 \%$ & & $56 \%$ & & $53 \%$ & \\
\hline IGF2 & $88 \%$ & & $85 \%$ & & $56 \%$ & & $87 \%$ & \\
\hline KDR & $69 \%$ & $0 \%$ & $54 \%$ & $6 \%$ & $31 \%$ & $0 \%$ & $40 \%$ & $0 \%$ \\
\hline KIT & & $0 \%$ & & $12 \%$ & & $0 \%$ & & $0 \%$ \\
\hline KRAS & & $0 \%$ & & $6 \%$ & & $11 \%$ & & $0 \%$ \\
\hline MDM2 & $81 \%$ & & $77 \%$ & & $50 \%$ & & $93 \%$ & \\
\hline MET & $69 \%$ & $0 \%$ & $62 \%$ & $12 \%$ & $31 \%$ & $5 \%$ & $60 \%$ & $0 \%$ \\
\hline MTOR & $81 \%$ & & $100 \%$ & & $44 \%$ & & $80 \%$ & \\
\hline NOTCH1 & & $0 \%$ & & $0 \%$ & & $0 \%$ & & $0 \%$ \\
\hline NRAS & & $0 \%$ & & $0 \%$ & & $0 \%$ & & $6 \%$ \\
\hline PIK3CA & & $0 \%$ & & $6 \%$ & & $0 \%$ & & $24 \%$ \\
\hline RET & & $6 \%$ & & $6 \%$ & & $5 \%$ & & $18 \%$ \\
\hline ТР53 & & $0 \%$ & & $0 \%$ & & $63 \%$ & & $65 \%$ \\
\hline
\end{tabular}

\section{DISCUSSION}

Until now, studies investigating novel therapeutic approaches for pulmonary carcinoid tumors are lacking due to the rather long overall survival rates of patients with carcinoids. Nevertheless, tumors associated with a syndrome are resistant to chemo- and radiotherapy in most cases. This study is one of the first approaches to investigate a broad spectrum of biomarkers potentially predicting response to different chemotherapeutic agents. For mRNA expression analysis, the NanoString nCounter technology was used and additionally, 221 mutation hotspots were screened via massive parallel sequencing by synthesis.

Erlotinib, gefitinib or icotinib, in second generation also afatinib, are epidermal growth factor receptor tyrosine-kinase inhibitors (EGFR-TKI) used for the treatment of NSCLC harboring activating EGFR mutations [28]. Pathway activation downstream of EGFR, e.g. by KRAS mutations, is known to be a resistance mechanism for EGFR-TKI therapeutic approaches, but also the appearance of resistance mutations within the EGFR gene (e.g. p.T790M) has been described [29]. Moreover, continued activation of PI3K signaling via mutated PIK3CA was identified to abrogate gefitinib-induced apoptosis [29]. Further studies confirmed that the PI3K pathway is involved in different resistance mechanisms with respect to EGFR therapy approaches [30]. Consequently, different trials using PI-103, a PI3K/mTOR double inhibitor, in combination with EGFR-TKI therapy were initiated.

In our study, none of the tumors showed classical activating mutations in the EGFR gene. Of note, eight mutations impairing protein function were found in pulmonary neuroendocrine tumors regardless of the subtype (figure 2). A present study revealed that also cases with other non-classical mutations and complex mutational events had similar end-point outcomes to TKI therapy compared with classical activating mutations [31]. Besides, anti-EGFR antibody therapy with cetuximab may be a possible alternative. Cetuximab activity in NSCLC was found 
in tumors that expressed high levels of EGFR only [32], but also other monoclonal anti-EGFR antibodies were evaluated. These include matuzumab, panitumumab and most promising necitumumab, leading to an increased survival compared to cetuximab [33]. In our study, the majority of carcinoid tumors and more than half of all high-grade tumors show strongly upregulated expression levels of EGFR; one third of all NELC present with a highly increased EGFR gene expression level (figure 2). Furthermore, it has been reported that more than half of all carcinoids show increased EGFR copy numbers [34]. Thus, making them potential targets for monoclonal anti-EGFR antibodies.

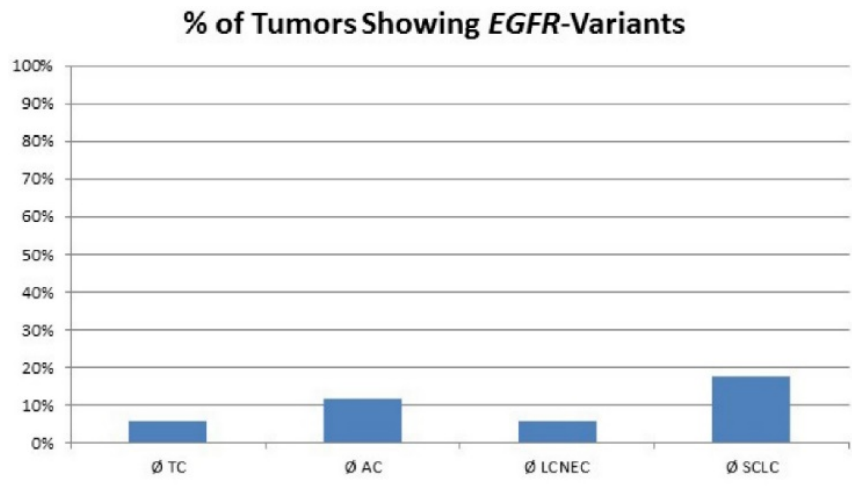

\% of Tumors Expressing EGFR

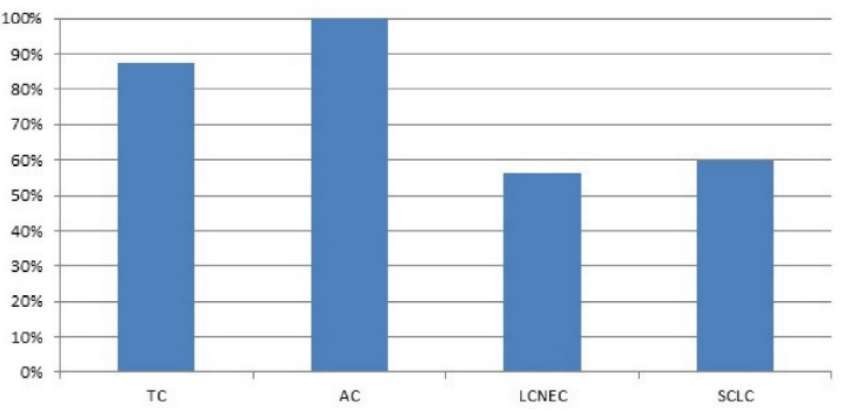

Figure 2. The plot in the upper row shows the percentage of tumours with EGFR variants predicted to influence protein function. Nevertheless, classical EGFR mutations were not found in the collective investigated. The plot at the bottom shows the percentage of tumours expressing EGFR. Carcinoids present with elevated EGFR expression compared to carcinomas.

Lapatinib is currently approved for patients with ERBB2 (HER2)-positive metastatic breast cancer in combination with capecitabine after prior therapy with anthracycline, taxane, and trastuzumab $[35,36]$. It is also approved in combination with letrozole in postmenopausal women with ERBB2-positive advanced breast cancer where endocrine therapy is indicated [36, 37]. Mutations in the ERBB2 (HER2) gene locus were reported in $1.5 \%-2 \%$ of all NSCLC, but this aberration occurred exclusively in adenocarcinomas [38, 39]. We found nine patients $(13 \%)$ harboring the activating p.L785R and the
p.R868W. It has been shown that patients with mutations and without amplification of the ERBB2 gene locus can respond to lapatinib [36, 39].

Activating BRAF mutations were found in two LCNEC and one SCLC. Patients harboring activating $B R A F$ mutations are known to be sensitive for therapy with vemurafenib [40]. Activation downstream of $B R A F$, primary through $R A S$ mutations, is reported to act as a potential resistance mechanism [41]. RAS mutations were observed in all tumor entities except $\mathrm{TC}$, but never appeared in combination with $B R A F$ mutations. The response of pulmonary neuroendocrine tumors harboring $B R A F$ mutations to vemurafenib has to be proven in further studies.

A large part of human cancers shows TP53 inactivation, mostly by mutations or loss of heterozygosity $(\mathrm{LOH})$ of the whole gene locus. Even in TP53 wild type tumors the functional protein is inactivated resulting from some putative mechanisms of amplification and overexpression of MDM2, the physiological inhibitor of P53 [42-46]. MDM2 itself is controlled by the tumor suppressor P14/ARF (encoded by the gene locus of CDKN2A). Numerous environmental influences and genetic alterations induced by platin compounds are able to activate P53 by post-translational modifications, which results in cell cycle arrest, cellular senescence, or apoptosis [47]. Nutlin-3A, a cis-imidazoline analogue, is a potent and selective MDM2 inhibitor [48, 49] that prevents MDM2-TP53-interaction by binding to the hydrophobic binding pocket of MDM2 leading to an immediate reactivation of P53 [48, 50, 51]. It is currently tested in a phase I clinical trial (NCT01143519, NCT00623870) [51]. Second-generation MDM2 inhibitors (RG7112, RO5045337, Idasanutlin, RG-7388, DS-3032b, SAR405838, CGM-097, MK8242, HDM201 etc.) are currently tested in phase I-III trials for various diseases (NCT01877382, NCT02319369, NCT00559533, NCT01985191, NCT02016729, NCT02343172 etc.)

For high-grade neuroendocrine lung tumors, TP53 mutations were found in the majority of tumors (figure 3A). As described, the main alternative mechanism seems to be $\mathrm{LOH}$, so MDM2 inhibition may not be successful. In contrast, carcinoids lack genetic alterations of TP53, but about $80 \%$ of samples show a dramatically high MDM2 gene expression level (figure 3B). Additionally, CDKN2A inhibiting MDM2 is absent in most pulmonary carcinoids. Therefore, pulmonary carcinoids seem to be an ideal target for Nutlin-3A therapy. A major issue with carcinoid tumors is the rarity of the disease and therefore it is difficult to conduct such studies. Also, there is an issue if drugs such as; everolimus could be administered as adjuvant therapy and for how long 
after surgery [52]. Everolimus could be used as treatment in the case that surgery is not possible, or could be used as adjuvant therapy in the case that surgery is not possible and only endoscopic debulking procedures are possible. The length of the therapy administration depends on multiple factors such as local disease progression and drug side effects. There are still many matters to clarify as disease diagnosis and management. [53-55]

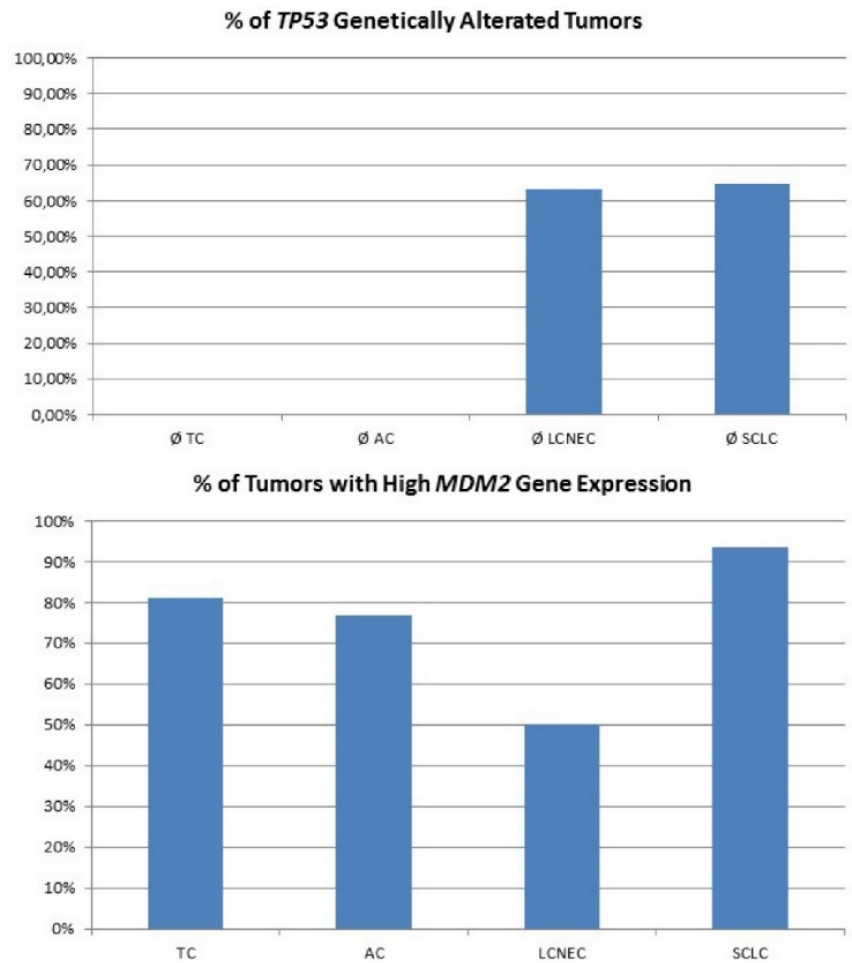

Figure 3. The plot at the top is showing the percentage of tumours with inactivating mutations in the TP53 gene. The plot at the bottom shows the percentage of tumours with high MDM2 expression. MDM2 overexpression is one possible mechanism to avoid TP53-dependet apoptosis and cell senescence.

\section{CONCLUSION}

Carcinoids of the lung show long survival rates, but sometimes progress to a systemic disease. Carcinoids with a syndrome are resistant to chemoand radiotherapy and new therapeutic approaches are needed. Based on our results, therapeutic approaches with MDM2 inhibitors and monoclonal anti-EGFR antibodies may be a promising novel therapeutic approach and need to be confirmed in further in vitro and in vivo studies.

\section{SUPPLEMENTARY MATERIAL}

Supplementary table 1.

http://www.jcancer.org/v07p2165s1.xlsx

\section{ACKNOWLEDGEMENTS}

We thank the nCounter Core Facility Heidelberg for providing the nCounter system and related services.

\section{CONFLICT OF INTEREST}

All authors disclose any affiliations that are considered to be relevant and important with any organization that to our knowledge has any direct interest in the subject matter discussed.

\section{REFERENCES}

1. Eramo A, Lotti F, Sette G, Pilozzi E, Biffoni M, Di Virgilio A, et al. Identification and expansion of the tumorigenic lung cancer stem cell population. Cell death and differentiation. 2008; 15: 504-14. doi: $10.1038 /$ sj.cdd. 4402283 .

2. Jett JR, Midthun DE. Screening for lung cancer: current status and future directions: Thomas A. Neff lecture. Chest. 2004; 125: 158S-62S.

3. Mallick R, Patnaik SK, Yendamuri S. MicroRNAs and lung cancer: Biology and applications in diagnosis and prognosis. Journal of carcinogenesis. 2010; 9. doi:10.4103/1477-3163.67074.

4. Rekhtman N. Neuroendocrine tumors of the lung: an update. Archives of pathology \& laboratory medicine. 2010; 134: 1628-38. doi:10.1043/2009-0583-RAR.1.

5. Takei H, Asamura H, Maeshima A, Suzuki K, Kondo H, Niki T, et al. Large cell neuroendocrine carcinoma of the lung: a clinicopathologic study of eighty-seven cases. The Journal of thoracic and cardiovascular surgery. 2002; 124: 285-92.

6. Thomas CF, Jr., Tazelaar HD, Jett JR. Typical and atypical pulmonary carcinoids: outcome in patients presenting with regional lymph node involvement. Chest. 2001; 119: 1143-50.

7. Travis WD, Rush W, Flieder DB, Falk R, Fleming MV, Gal AA, et al. Survival analysis of 200 pulmonary neuroendocrine tumors with clarification of criteria for atypical carcinoid and its separation from typical carcinoid. The American journal of surgical pathology. 1998; 22: 934-44.

8. Asamura H, Kameya T, Matsuno $Y$, Noguchi M, Tada H, Ishikawa Y, et al. Neuroendocrine neoplasms of the lung: a prognostic spectrum. Journal of clinical oncology: official journal of the American Society of Clinical Oncology. 2006; 24: 70-6. doi:10.1200/JCO.2005.04.1202.

9. Cardillo G, Sera F, Di Martino M, Graziano P, Giunti R, Carbone L, et al. Bronchial carcinoid tumors: nodal status and long-term survival after resection. The Annals of thoracic surgery. 2004; 77: 1781-5. doi:10.1016/j.athoracsur.2003.10.089.

10. Beasley MB, Thunnissen FB, Brambilla E, Hasleton P, Steele R, Hammar SP, et al. Pulmonary atypical carcinoid: predictors of survival in 106 cases. Human pathology. 2000; 31: 1255-65. doi:10.1053/hupa.2000.19294.

11. Fink G, Krelbaum T, Yellin A, Bendayan D, Saute M, Glazer M, et al. Pulmonary carcinoid: presentation, diagnosis, and outcome in 142 cases in Israel and review of 640 cases from the literature. Chest. 2001; 119: 1647-51.

12. Garcia-Yuste M, Matilla JM, Cueto A, Paniagua JM, Ramos G, Canizares MA, et al. Typical and atypical carcinoid tumours: analysis of the experience of the Spanish Multi-centric Study of Neuroendocrine Tumours of the Lung. European journal of cardio-thoracic surgery: official journal of the European Association for Cardio-thoracic Surgery. 2007; 31: 192-7. doi:10.1016/j.ejcts.2006.11.031.

13. Pelosi G, Scarpa A, Puppa G, Veronesi G, Spaggiari L, Pasini F, et al. Alteration of the E-cadherin/beta-catenin cell adhesion system is common in pulmonary neuroendocrine tumors and is an independent predictor of lymph node metastasis in atypical carcinoids. Cancer. 2005; 103: 1154-64. doi:10.1002/cncr.20901.

14. Rea F, Rizzardi G, Zuin A, Marulli G, Nicotra S, Bulf R, et al. Outcome and surgical strategy in bronchial carcinoid tumors: single institution experience with 252 patients. European journal of cardio-thoracic surgery: official journal of the European Association for Cardio-thoracic Surgery. 2007; 31: 186-91. doi:10.1016/j.ejcts.2006.10.040.

15. Travis WD. Lung tumours with neuroendocrine differentiation. Eur J Cancer. 2009; 45 Suppl 1: 251-66. doi:10.1016/S0959-8049(09)70040-1.

16. Scott WJ. Surgical treatment of other bronchial tumors. Chest surgery clinics of North America. 2003; 13: 111-28.

17. Swarts DR, Ramaekers FC, Speel EJ. Molecular and cellular biology of neuroendocrine lung tumors: evidence for separate biological entities. $\begin{array}{llll}\text { Biochimica et biophysica acta. 2012; 1826: 255-71. } & \end{array}$ doi:10.1016/j.bbcan.2012.05.001

18. Travis WD, World Health Organization., International Agency for Research on Cancer., International Association for the Study of Lung Cancer., International Academy of Pathology. Pathology and genetics of tumours of the lung, pleura, thymus and heart. Oxford: IARC Press. 2004. 
19. Gridelli C, Rossi A, Airoma G, Bianco R, Costanzo R, Daniele B, et al Treatment of pulmonary neuroendocrine tumours: State of the art and future developments. Cancer treatment reviews. 2012. doi:10.1016/j.ctrv.2012.06.012.

20. Gridelli C, Rossi A, Airoma G, Bianco R, Costanzo R, Daniele B, et al. Treatment of pulmonary neuroendocrine tumours: State of the art and future developments. Cancer treatment reviews. 2013; 39: 466-72. doi:10.1016/j.ctrv.2012.06.012.

21. Mackley HB, Videtic GM. Primary carcinoid tumors of the lung: a role for radiotherapy. Oncology (Williston Park). 2006; 20: 1537-43; discussion 44-5, 49.

22. Rossi G, Cavazza A, Marchioni A, Longo L, Migaldi M, Sartori G, et al. Role of chemotherapy and the receptor tyrosine kinases KIT, PDGFRalpha, PDGFRbeta, and Met in large-cell neuroendocrine carcinoma of the lung. Journal of clinical oncology: official journal of the American Society of Clinical Oncology. 2005; 23: 8774-85. doi:10.1200/JCO.2005.02.8233.

23. Reva B, Antipin Y, Sander C. Predicting the functional impact of protein mutations: application to cancer genomics. Nucleic acids research. 2011; 39: e118. doi:10.1093/nar/gkr407.

24. Wang K, Li M, Hakonarson $\mathrm{H}$. ANNOVAR: functional annotation of genetic variants from high-throughput sequencing data. Nucleic acids research. 2010; 38: e164. doi:10.1093/nar/gkq603.

25. Kumar P, Henikoff S, Ng PC. Predicting the effects of coding non-synonymous variants on protein function using the SIFT algorithm. Nature protocols. 2009; 4: 1073-81. doi:10.1038/nprot.2009.86.

26. Adzhubei IA, Schmidt S, Peshkin L, Ramensky VE, Gerasimova A, Bork P, et al. A method and server for predicting damaging missense mutations. Nature methods. 2010; 7: 248-9. doi:10.1038/nmeth0410-248.

27. Schwarz JM, Rodelsperger C, Schuelke M, Seelow D. MutationTaster evaluates disease-causing potential of sequence alterations. Nature methods. 2010; 7: 575-6. doi:10.1038/nmeth0810-575.

28. Sarosi V, Losonczy G, Francovszky E, Tolnay E, Torok S, Galffy G, et al. Effectiveness of erlotinib treatment in advanced KRAS mutation-negative lung adenocarcinoma patients: Results of a multicenter observational cohort study (MOTIVATE). Lung Cancer. 2014. doi:10.1016/j.lungcan.2014.07.011.

29. Engelman JA, Mukohara T, Zejnullahu K, Lifshits E, Borras AM, Gale CM, et al. Allelic dilution obscures detection of a biologically significant resistance mutation in EGFR-amplified lung cancer. The Journal of clinical investigation. 2006; 116: 2695-706. doi:10.1172/JCI28656.

30. Sequist LV, Waltman BA, Dias-Santagata D, Digumarthy $S$, Turke AB, Fidias $\mathrm{P}$, et al. Genotypic and histological evolution of lung cancers acquiring resistance to EGFR inhibitors. Science translational medicine. 2011; 3: 75ra26. doi:10.1126/scitranslmed.3002003.

31. Peng L, Song ZG, Jiao SC. Efficacy analysis of tyrosine kinase inhibitors on rare non-small cell lung cancer patients harboring complex EGFR mutations. Scientific reports. 2014; 4: 6104. doi:10.1038/srep06104.

32. Amendt $C$, Staub E, Friese-Hamim M, Storkel S, Stroh C. Association of EGFR expression level and cetuximab activity in patient-derived xenograft models of human non-small cell lung cancer. Clinical cancer research: an official journal of the American Association for Cancer Research. 2014. doi:10.1158/1078-0432.CCR-13-3385.

33. Pirker R. EGFR-directed monoclonal antibodies in non-small cell lung cancer. Targeted oncology. 2013; 8: 47-53. doi:10.1007/s11523-012-0244-7.

34. Gilbert JA, Adhikari LJ, Lloyd RV, Rubin J, Haluska P, Carboni JM, et al Molecular markers for novel therapies in neuroendocrine (carcinoid) tumors. Endocrine-related cancer. 2010; 17: 623-36. doi:10.1677/ERC-09-0318.

35. Geyer CE, Forster J, Lindquist D, Chan S, Romieu CG, Pienkowski T, et al. Lapatinib plus capecitabine for HER2-positive advanced breast cancer. The New England journal of medicine. 2006; 355: 2733-43. doi:10.1056/NEJMoa064320.

36. Tan AR, Dowlati A, Stein MN, Jones SF, Infante JR, Bendell J, et al. Phase I study of weekly paclitaxel in combination with pazopanib and lapatinib in advanced solid malignancies. British journal of cancer. 2014; 110: 2647-54. doi:10.1038/bjc.2014.233

37. Johnston S, Pippen J, Jr., Pivot X, Lichinitser M, Sadeghi S, Dieras V, et al. Lapatinib combined with letrozole versus letrozole and placebo as first-line therapy for postmenopausal hormone receptor-positive metastatic breast cancer. Journal of clinical oncology: official journal of the American Society of Clinical Oncology. 2009; 27: 5538-46. doi:10.1200/JCO.2009.23.3734.

38. Mazieres J, Peters S, Lepage B, Cortot AB, Barlesi F, Beau-Faller M, et al. Lung cancer that harbors an HER2 mutation: epidemiologic characteristics and therapeutic perspectives. Journal of clinical oncology: official journal of the American Society of Clinical Oncology. 2013; 31: 1997-2003. doi:10.1200/JCO.2012.45.6095.

39. Rolfo C, Giovannetti E, Hong DS, Bivona T, Raez LE, Bronte G, et al. Novel therapeutic strategies for patients with NSCLC that do not respond to treatment with EGFR inhibitors. Cancer treatment reviews. 2014; 40: 990-1004. doi:10.1016/j.ctrv.2014.05.009.

40. Kim G, McKee AE, Ning YM, Hazarika M, Theoret M, Johnson JR, et al. FDA Approval Summary: Vemurafenib for Treatment of Unresectable or Metastatic Melanoma with the BRAF V600E Mutation. Clinical cancer research: an official journal of the American Association for Cancer Research. 2014. doi:10.1158/1078-0432.CCR-14-0776

41. Sos ML, Levin RS, Gordan JD, Oses-Prieto JA, Webber JT, Salt M, et al. Oncogene Mimicry as a Mechanism of Primary Resistance to BRAF Inhibitors. Cell reports. 2014. doi:10.1016/j.celrep.2014.07.010
42. Jones $\mathrm{SN}$, Roe AE, Donehower LA, Bradley A. Rescue of embryonic lethality in Mdm2-deficient mice by absence of p53. Nature. 1995; 378: 206-8. doi:10.1038/378206a0.

43. Marine JC, Francoz S, Maetens M, Wahl G, Toledo F, Lozano G. Keeping p53 in check: essential and synergistic functions of Mdm2 and Mdm4. Cell death and differentiation. 2006; 13: 927-34. doi:10.1038/sj.cdd.4401912.

44. Montes de Oca Luna R, Wagner DS, Lozano G. Rescue of early embryonic lethality in mdm2-deficient mice by deletion of p53. Nature. 1995; 378: 203-6. doi:10.1038/378203a0.

45. Parant J, Chavez-Reyes A, Little NA, Yan W, Reinke V, Jochemsen AG, et al. Rescue of embryonic lethality in Mdm4-null mice by loss of Trp53 suggests a nonoverlapping pathway with MDM2 to regulate p53. Nature genetics. 2001; 29: 92-5. doi:10.1038/ng714.

46. Ringshausen I, O'Shea CC, Finch AJ, Swigart LB, Evan GI. Mdm2 is critically and continuously required to suppress lethal p53 activity in vivo. Cancer cell. 2006; 10: 501-14. doi:10.1016/j.ccr.2006.10.010.

47. Harris SL, Levine AJ. The p53 pathway: positive and negative feedback loops. Oncogene. 2005; 24: 2899-908. doi:10.1038/sj.onc.1208615.

48. Vassilev LT, Vu BT, Graves B, Carvajal D, Podlaski F, Filipovic Z, et al. In vivo activation of the p53 pathway by small-molecule antagonists of MDM2. Science. 2004; 303: 844-8. doi:10.1126/science.1092472.

49. Shangary S, Wang S. Small-molecule inhibitors of the MDM2-p53 protein-protein interaction to reactivate p53 function: a novel approach for cancer therapy. Annual review of pharmacology and toxicology. 2009; 49: 223-41. doi:10.1146/annurev.pharmtox.48.113006.094723.

50. Gamble LD, Kees UR, Tweddle DA, Lunec J. MYCN sensitizes neuroblastoma to the MDM2-p53 antagonists Nutlin-3 and MI-63. Oncogene. 2012; 31: 752-63. doi:10.1038/onc.2011.270.

51. Voltan R, Secchiero P, Ruozi B, Forni F, Agostinis C, Caruso L, et al. Nanoparticles engineered with rituximab and loaded with Nutlin-3 show promising therapeutic activity in B-leukemic xenografts. Clinical cancer research : an official journal of the American Association for Cancer Research. 2013; 19: 3871-80. doi:10.1158/1078-0432.CCR-13-0015.

52. Flaum N, Valle JW, Mansoor W, McNamara MG. Everolimus in the treatment of neuroendocrine tumors of the respiratory and gastroenteropancreatic systems. Future oncology. 2016. doi:10.2217/fon.16.23.

53. Wolin EM. Advances in the Diagnosis and Management of Well-Differentiated and Intermediate-Differentiated Neuroendocrine Tumors of the Lung. Chest. 2016. doi:10.1016/j.chest.2016.06.018.

54. Cives M, Soares HP, Strosberg J. Will clinical heterogeneity of neuroendocrine tumors impact their management in the future? Lessons from recent trials. Current opinion in oncology. 2016; 28: 359-66. doi:10.1097/CCO.0000000000000299.

55. Rugo HS, Hortobagyi GN, Yao J, Pavel M, Ravaud A, Franz D, et al. Meta-analysis of stomatitis in clinical studies of everolimus: incidence and relationship with efficacy. Annals of oncology: official journal of the European Society for Medical Oncology / ESMO. 2016; 27: 519-25. doi:10.1093/annonc/mdv595. 